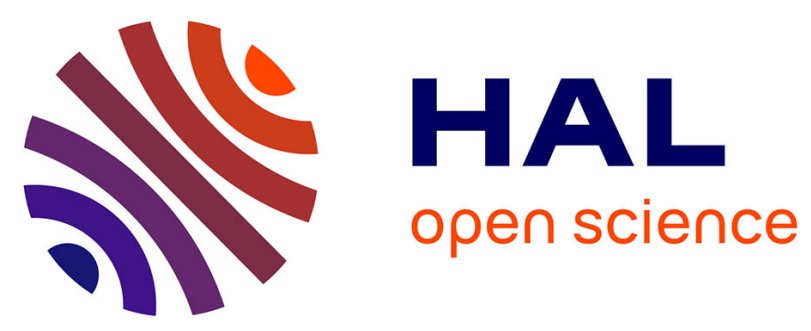

\title{
Uniaxial magnetic anisotropy induced by vicinal surfaces in half metallic La0.7Sr0.3MnO3 thin films
}

Paolo Perna, E. Jiménez, F. J. Teran, Laurence Méchin, Julio Camarero, R. Miranda

\section{- To cite this version:}

Paolo Perna, E. Jiménez, F. J. Teran, Laurence Méchin, Julio Camarero, et al.. Uniaxial magnetic anisotropy induced by vicinal surfaces in half metallic La0.7Sr0.3MnO3 thin films. MRS Fall Meeting, Nov 2010, Boston, United States. pp.1198-E01-04. hal-00976876

\section{HAL Id: hal-00976876 https://hal.science/hal-00976876}

Submitted on 10 Apr 2014

HAL is a multi-disciplinary open access archive for the deposit and dissemination of scientific research documents, whether they are published or not. The documents may come from teaching and research institutions in France or abroad, or from public or private research centers.
L'archive ouverte pluridisciplinaire HAL, est destinée au dépôt et à la diffusion de documents scientifiques de niveau recherche, publiés ou non, émanant des établissements d'enseignement et de recherche français ou étrangers, des laboratoires publics ou privés. 


\title{
Uniaxial magnetic anisotropy induced by vicinal surfaces in half metallic $\mathrm{La}_{0.7} \mathrm{Sr}_{0.3} \mathrm{MnO}_{3}$ thin films
}

\author{
P. Perna ${ }^{1}$, E. Jiménez ${ }^{2}$, F. J. Terán ${ }^{1}$, L. Méchin ${ }^{3}$, J. Camarero ${ }^{1,2}$ and R. Miranda ${ }^{1,2}$ \\ ${ }^{1}$ Instituto Madrileño de Estudios Avanzados en Nanociencia IMDEA-Nanociencia, Campus \\ Universidad Autónoma de Madrid, 28049 Madrid, Spain \\ 2 Departamento de Física de la Materia Condensada and Instituto "Nicolás Cabrera", \\ Universidad Autónoma de Madrid, 28049 Madrid, Spain \\ ${ }^{3}$ GREYC-ENSICAEN and Université de Caen-Basse Normandie, Bd. de Marèchal Juan, 14050 \\ Caen, France
}

\begin{abstract}
We present a detailed study of the angular dependence of the magnetization reversal at room temperature of well characterized epitaxial $\mathrm{La}_{0.7} \mathrm{Sr}_{0.3} \mathrm{MnO}_{3}(001)$ thin films grown onto $\mathrm{SrTiO}_{3}$ (001) vicinal substrates. The step edges at the substrate surface promote a topological modulation of the films along the step direction, breaking the four-fold magneto crystalline symmetry and favoring a two-fold magnetic anisotropy term. The competition between the biaxial and uniaxial anisotropy is depicted within the framework of the current theory, resulting in a vanishing biaxial contribution. The films hence show the magnetization easy (hard) direction parallel (perpendicular) to the steps direction. The thickness-dependent of both anisotropy and magnetization reversal are discussed in terms of topographic changes.
\end{abstract}

\section{INTRODUCTION}

The magnetic properties of half-metallic ferromagnetic $\mathrm{La}_{0.7} \mathrm{Sr}_{0.3} \mathrm{MnO}_{3}$ (LSMO) films are known to be very sensitive to both crystallographic and morphological variations [1-4]. For (001)-oriented LSMO the strain imposed by the lattice mismatch between the film and the substrate can determine the magnetization easy-axis orientation, i.e., magnetic anisotropy. In general, in-plane compressive strain enhances out-of-plane magnetization, whereas in-plane tensile strain increases the in-plane magnetization component. In addition, preferential morphology shapes, produced by patterning or by using vicinal substrates, can also alter their magnetic anisotropy. All these concern to both magnetization easy and hard axis directions as well as the magnetization reversal processes [5].

In LSMO thin films deposited on (001) $\mathrm{SrTiO}_{3}$ (STO) substrates can compete three in-plane magnetic anisotropy terms: magnetocrystalline biaxial anisotropy (four-fold symmetry), originating from tensile strain, uniaxial magnetic anisotropy (two-fold symmetry), originating from a preferential substrate step orientation, and shape anisotropy, in the case of patterned nanostructures. The biaxial anisotropy present the easy in-plane direction along [110] and the hard in-plane direction along $[100][4,6,7,8,9,10]$ where as the easy-axis of the uniaxial anisotropy lies parallel to the substrate step edge direction [11-14]. This uniaxial contribution dominates at room temperature in LSMO (100) films grown on very low miscut STO (100) substrates $\left(0.13^{\circ}\right.$ and $\left.0.24^{\circ}\right)$ [11], which vanishes at low temperature where biaxial 
magnetocrystalline anisotropy dominates $(160 \mathrm{~K})$. It has also been found that the uniaxial term is still dominant at $80 \mathrm{~K}$ by using higher miscut STO substrates $\left(10^{\circ}\right)$ [14].

In this work, we present a detailed angular study of the magnetization reversal performed at room temperature of well characterized epitaxial LSMO (001) thin films grown by pulsed laser deposition onto STO (001) vicinal substrates $\left(10^{\circ}\right.$ miscut $)$. The films show only in-plane uniaxial magnetic anisotropy with the magnetization easy-axis oriented parallel to the substrate step edge direction. Both magnetization reversal and anisotropy field depend on the LSMO thickness, and are related with the film topography.

\section{EXPERIMENT}

The LSMO thin films were deposited by pulsed laser deposition from a stoichiometric target onto commercially available vicinal STO (001) substrates at different thickness (namely 15, 70 $\mathrm{nm}$ ) with vicinal angle of $10^{\circ}$ from the [001] direction towards [110], thus inducing steps along the [110] crystallographic directions (see sketch in Figure 1 A). The optimization of the growth conditions was performed on standard STO (001) substrates [15]. The laser fluence was $1-2 \mathrm{Jcm}^{-}$ ${ }^{2}$, the target-to-substrate distance was $50 \mathrm{~mm}$, the oxygen pressure was $0.35 \mathrm{mbar}$ and the substrate temperature was kept at $720^{\circ} \mathrm{C}$. Resistivity and magnetization measurements performed in the two films investigated reveal a similar Curie temperature of about $350 \mathrm{~K}$ (not shown).

The X-Ray Diffraction (XRD) measurements proved the high crystalline quality and the epitaxy of the LSMO films. Offset angle of the LSMO film was checked to be equal to the substrate vicinal angle within $0.05^{\circ} . \theta-2 \theta$ scans (Figure $1 \mathrm{~B}$ ) allow us to determine the out-ofplane lattice parameters of the samples. The out-of-plane lattice parameters of the $15 \mathrm{~nm}$ and 70 $\mathrm{nm}$ thick vicinal LSMO films are $0.3850(3) \mathrm{nm}$ and $0.3851(3) \mathrm{nm}$, respectively, which correspond to a lattice mismatch with STO of about $1.4 \times 10^{-2}$ [13]. For both thicknesses, the LSMO films are fully tensile in-plane strained onto the substrate showing in-plane lattice parameters of $0.3905(3) \mathrm{nm}$, i.e. equal to one of the substrate, as demonstrated by XRD measurements mapping regions around the asymmetric crystallographic film peaks (not shown). Moreover, the presence of the interference fringes at the side of the LSMO (002) crystallographic peak is an indication of the high surface quality and low roughness of the films. The full-width-at-half-maximum of the rocking curve around the (002) peak of the vicinal LSMO films was $0.23^{\circ}$, which is comparable to typical values obtained in $75 \mathrm{~nm}$ thick LSMO films deposited on nominally flat (001)-oriented STO substrates. 
A.

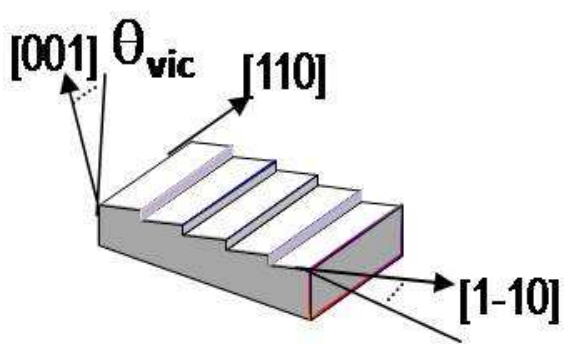

C.

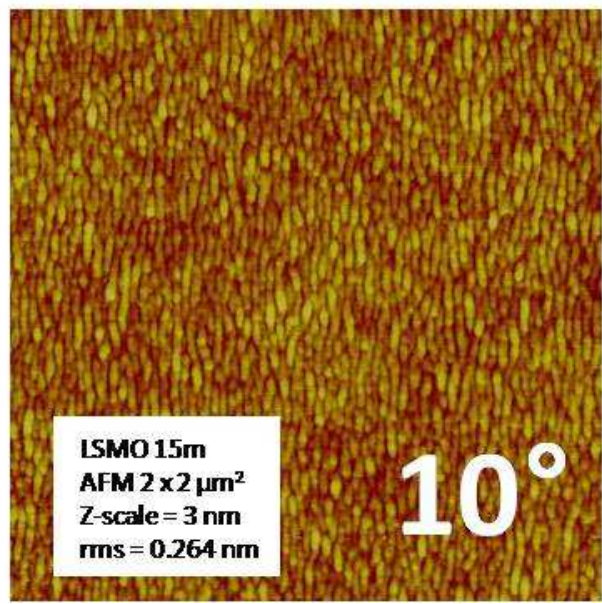

B.

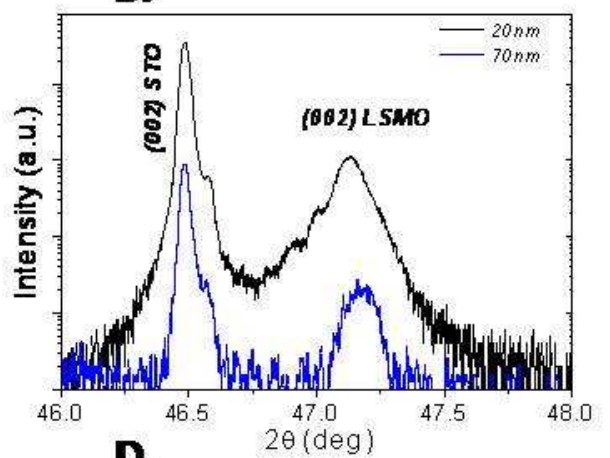

D.

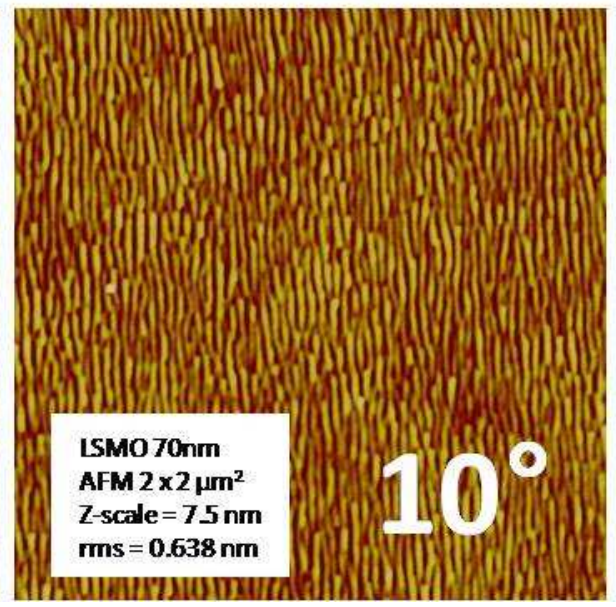

Figure 1. (color) (A) Sketch of the vicinal $10^{\circ}$ STO surface. (B) XRD $\theta-2 \theta$ scans around the (002) STO peak of the LSMO 70nm thick (black curve) and $15 \mathrm{~nm}$ thick (blue curve) grown onto $10^{\circ}$ vicinal (001) STO substrates. Atomic force microscopy (AFM) of the top surface of the LSMO films $15 \mathrm{~nm}(\mathrm{C})$ and $70 \mathrm{~nm}(\mathrm{D})$ thick grown onto (001) STO $10^{\circ}$ vicinal substrates.

Atomic force and scanning probe microscopies were performed in order to investigate the morphology of the samples. The average roughness (RMS) of the samples, determined by means of AFM, was 1-2 unit cells (u.c). As shown in Figure 1, the LSMO films present elongated structures running parallel to the substrate step edge direction, i.e., [1-10], for both investigated thickness $(15 \mathrm{~nm}$ and $70 \mathrm{~nm})$. A large aspect ratio is observed for the thinner film, which will be interpreted, in the following section, in term of magnetization reversal. In particular, such as elongated grains are found longer and better defined for thinner film (Figure $1 \mathrm{C}$ and D).

The angular dependence of the magnetization reversal was performed at room temperature (below $\mathrm{T}_{\mathrm{C}}$ ) by using our home-made high-resolution vectorial Kerr magnetometer. In our setup [5] the combination of p-polarized incident light in Kerr experiments and the simultaneous detection of the two orthogonal components of the reflected light allow the simultaneous determination of the components of the in-plane magnetization, parallel $(\mathrm{M} \|)$ and perpendicular $(\mathrm{M} \perp)$ to the field direction [16]. $\mathrm{M}_{\|}$originates from the difference of the two components of the reflected light, i.e., Kerr rotation, where as $\mathrm{M} \perp$ originates from the small variation of their sum, i.e., reflectivity changes. In-plane resolved hysteresis loops were obtained by averaging many successive iterations, with an acquisition time of $0.25 \mathrm{~s} / \mathrm{sweep}$. The study of the magnetization reversal processes and magnetic anisotropy of the films was performed at room temperature by 
measuring the in-plane resolved $\mathrm{M}-\mathrm{H}$ hysteresis loops as functions of the in-plane angular rotation $\theta$ in the whole angular range every $4.5^{\circ}$ with $0.5^{\circ}$ angular resolution.

\section{DISCUSSION}

Figure $2 \mathrm{~A}$ and $\mathrm{B}$ show the Kerr hysteresis loops recorded at selected angles $\theta$ between the magnetic field and the substrate step edge direction, i.e., [110] in-plane crystal direction for the two films with different thicknesses. In general, at $\theta=0^{\circ}$, i.e, parallel to the step edge direction, the squared shape of the $\mathrm{M}_{\|}(\mathrm{H})$ loop, just showing one irreversible sharp transition, and $\mathrm{M} \perp \mathrm{H})=0$ are signatures of a magnetization easy-axis direction. The hard-axis direction is found at $\theta=90^{\circ}$, i.e., perpendicular to the step edge direction, as shown in the bottom graphs of Figure 2 where large $\mathrm{M} \perp$ values and almost a non-hysteretic $\mathrm{M}_{\|}$behavior are observed. This corresponds with a magnetization reversal governed by coherent reversal processes, as expected for a hard axis direction. This uniaxial anisotropy can be also evidenced from the angular plots of both remanence magnetization, i.e., $\mathrm{M}_{\|, \mathrm{R}}$ and $\mathrm{M}_{\perp_{, \mathrm{R}}}$, as shown Figure 3 , where the values are reproduced every $180^{\circ}$, reflecting the two-fold symmetry.

The strength of the uniaxial anisotropy depends on the sample surface morphology. The coercive field $\left(\mu_{0} \mathrm{H}_{\mathrm{C}}\right)$ and anisotropy field $\left(\mu_{0} \mathrm{H}_{\mathrm{K}}\right)$ values decreases when the film thickness decreases. In addition, the hard axis $\mathrm{M}_{\|}(\mathrm{H})$ curve is more open for the thinner film (see Figure 3). This can also be observed in the angular plots of $\mathrm{M}_{\|, \mathrm{R}}$, where for the thinner film does not reach the zero value at the hard axis, as well as the $70 \mathrm{~nm}$ thick film. This indicates that magnetization reversal there is also a contribution of the nucleation and propagation of the domain walls. It is ascribed to the different morphology of the sample surfaces. In particular, the thickest film presents longer elongated grains compared to the thinnest one, as it can be seen in Figure $1 \mathrm{C}$ and $\mathrm{D}$.

In order to understand the physical nature of the magnetic anisotropy in our samples, we refer to the model depicted in Ref. [5] based on the coherent rotation model including biaxial (four-fold symmetry) and uniaxial (two-fold) magnetic anisotropy terms, which deals with the tensile-strain of the LSMO/STO system (magnetocrystalline) and the preferential substrate step orientation (shape), as discussed in the introduction, respectively. The model can reproduce the experimental data with a negligible biaxial term, indicating that in our films the dominant contribution to the magnetic anisotropy is originated from the substrate step orientation. 
A.

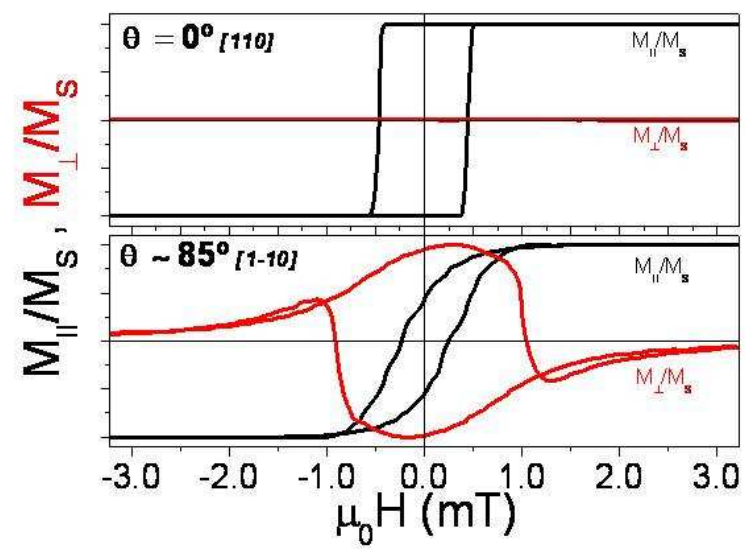

B.

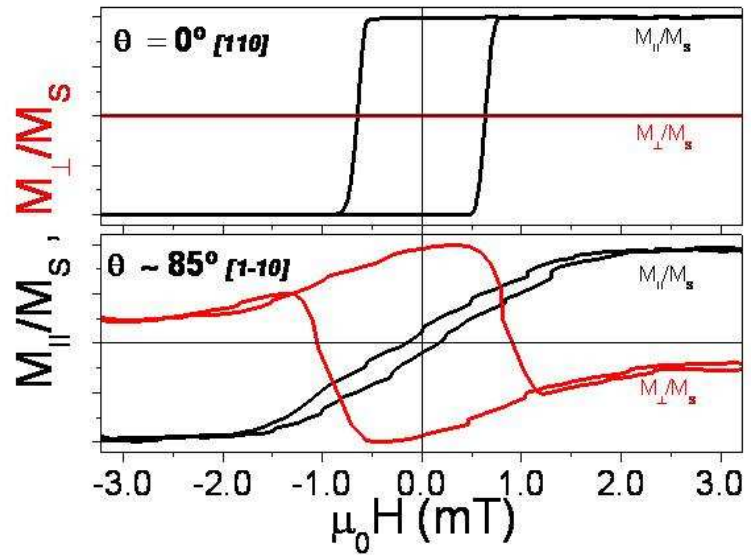

Figure 2. (color) $M_{\|}$and $M \perp$ normalized to the saturation magnetization $\left(M_{S}\right)$ around the e.a. $\left(\theta=0^{\circ} / /[110]\right)$ and close to h.a. $\left(\theta \sim 85^{\circ} / /[1-10]\right)$ for LSMO $15 \mathrm{~nm}$ thick (A) and $70 \mathrm{~nm}$ thick (B) films.

A.

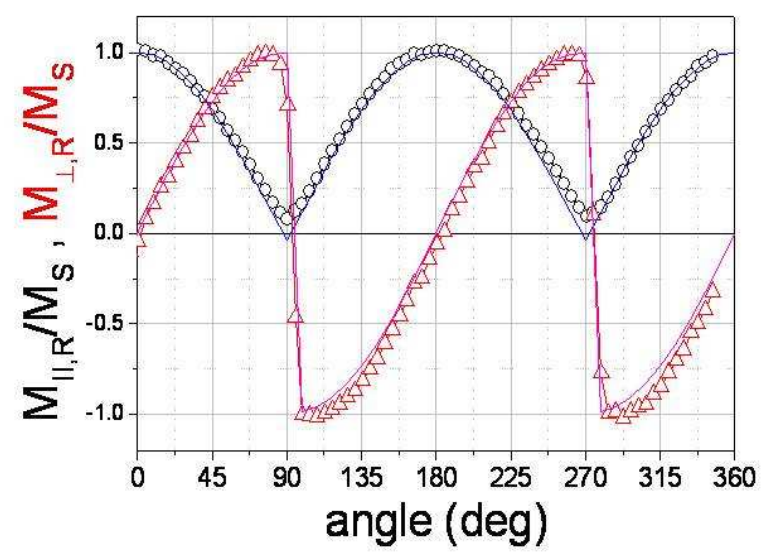

B.

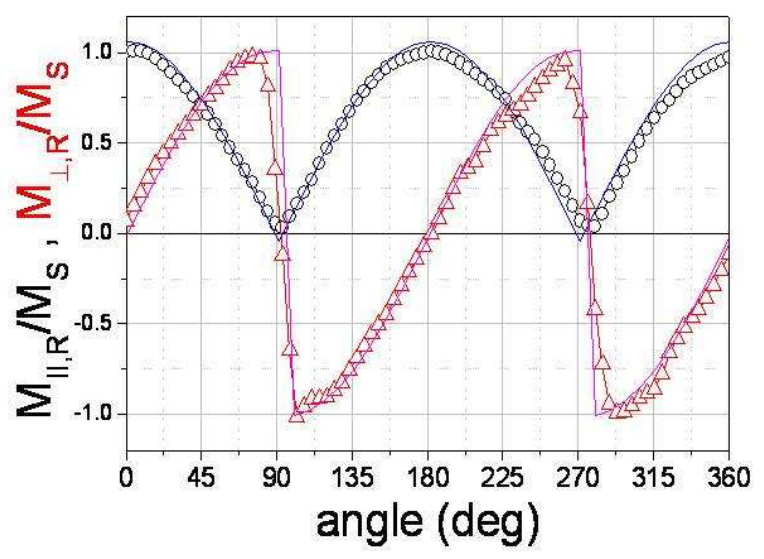

Figure 3. (color) Angular dependence of the reduced remanence components for LSMO 15nm thick (A) and 70nm thick (B) films. The open symbols are the experimental data, while lines are the numerical simulations extracted from the rotation model including only the uniaxial anisotropy term.

\section{CONCLUSIONS}

In conclusion, we have investigated the magnetic anisotropy of vicinal LSMO thin films by high-resolution vectorial Kerr magnetometer. The dominance of the uniaxial anisotropy over the biaxial anisotropy is achieved in LSMO thin films grown onto $10^{\circ}$ vicinal STO substrate. In such a system, the in-plane steps along the [110] crystallographic direction determine an easy axis for 
the magnetization parallel to the steps. In contrary, the [1-10] crystallographic direction (i.e. perpendicular to the steps) is the hard axis for the magnetization.

Exploiting to the presence of step edge at the vicinal substrate surface we are able to control and tailor the magnetic anisotropy of LSMO films. This result to be an important task for the design of novel planar devices based on thin film technology. It is worth to note that the use of relatively thick LSMO films (up to $70 \mathrm{~nm}$ ) is highly desirable for devices fabrication, since the magnetization value will be directly related to the ferromagnetic volume involved. Published data on vicinal LSMO films so far had concerned ultrathin films only (12-25 nm) [14].

\section{ACKNOWLEDGMENTS}

This work was supported in part by the Spanish MICINN through Project Nos. MAT200613470, MAT2007-66309-C02, and CSD2007-00010 and by the Comunidad de Madrid through Project No. S2009/MAT-1726.

\section{REFERENCES}

1. A. Ruotolo, A. Oropallo, F. Miletto Granozio, G.P. Pepe, P. Perna, U. Scotti di Uccio, and D. Pullini, Appl. Phys. Lett. 91, 132502 (2007)

2. M. Radovic, N. Lampis, F. Miletto Granozio, P. Perna, Z. Ristic, M. Salluzzo, C. M. Schleputz, and U. Scotti di Uccio, Appl. Phys. Lett. 94, 022901 (2009)

3. A. Ruotolo, A. Oropallo, F. Miletto Granozio, P. Perna and U. Scotti di Uccio, Appl. Phys. Lett. 88, 252504 (2006)

4. F. Tsui, M.C. Smoak, T.K. Nath, C.B. Eom, Appl. Phys. Lett. 76, 172421 (2000)

5. D. Ecija, E. Jiménez, N. Mikuszeit, N. Sacristán, J. Camarero. J. M. Gallego, J. Vogel and R. Miranda, Phys. Rev. B 77, 024426 (2008)

6. M. Bowen, M. Bibes, A. Barthelemy, J.-P. Contour, A. Anane, Y. Lemaitre and A. Fert, Appl. Phys. Lett. 82(2), 233 (2003)

7. K. Steenbeck, R. Hiergeist, Appl. Phys. Lett. 75(12), 1778 (1999)

8. P. Lecoeur, P.L. Trouilloud, G. Xiao, A. Gupta, G.Q. Gong, X.W. Li, J. Appl. Phys. 82(8), 3934

9. Suzuki, H.Y. Hwang, S-W. Cheong, R.B.van Dover, Appl. Phys. Lett. 71,(1) 140 (1997)

10. L.M. Berndt, V. Balbarin, Y. Suzuki, Appl. Phys. Lett. 77(18), 2903 (2000)

11. M. Mathews, F. M. Postma, J. C. Lodder, R. Jansen, G, Rijnders, and D. H. Blank, Appl. Phys. Lett. 87, 242507 (2005)

12. H.-U. Habermeier, J. Electrochem. 13, 23 (2004)

13. R.A. Hyman, A. Zangwill, M.D. Stiles, Phys. Rev. B 58(14), 9276 (1998)

14. Z.-H. Wang, G. Cristiani, H.-U. Habermeier, Appl. Phys. Lett. 82(21), 3731 (2003)

15. P. Perna, L. Méchin, M.P. Chauvat, P. Ruterana, Ch. Simon, and U. Scotti di Uccio, J. Phys.: Condens. Matter 21, 306005 (2009)

16. J. Camarero, J. Sort, A. Hoffmann, J. M. García-Martín, B. Dieny, R. Miranda, and J. Nogués, Phys. Rev. Lett. 95, 057204 (2005) 